\title{
Utility of Self-rated Adherence and Assessment of Participant Feedback of the Healthy Diet and Lifestyle Study Pilot.
}

Holly O’Reilly

Technological University Dublin

Chloe Panizza ( $\sim$ cpanizza@hawaii.edu)

University of Hawai'i Cancer Center https://orcid.org/0000-0003-4278-1602

Unhee Lim

University of Hawai'i Cancer Center

Kim M Yonemori

University of Hawai'i Cancer Center

Lynne R Wilkens

University of Hawai'i Cancer Center

Yurii B Shvetsov

University of Hawai'i Cancer Center

Michelle N Harvie

Manchester University NHS Foundation Trust

John Shepherd

University of Hawai'i Cancer Center

Fengqing Maggie Zhu

Purdue University

Loïc Le Marchand

University of Hawai'i Cancer Center

Carol J Boushey

University of Hawai'i Cancer Center

Kevin D Cassel

University of Hawai'i Cancer Center

\section{Research}

Keywords: Qualitative assessment, randomized controlled trial, self-rated adherence, visceral adipose tissue, weight loss.

Posted Date: August 5th, 2020 
DOI: https://doi.org/10.21203/rs.3.rs-52340/v1

License: (c) (i) This work is licensed under a Creative Commons Attribution 4.0 International License. Read Full License 


\section{Abstract}

Background: Self-rated adherence and qualitative feedback may be useful in monitoring and understanding participants' compliance during lifestyle interventions. We examined self-rated adherence and qualitative feedback in the Healthy Diet and Lifestyle Study (HDLS). HDLS is a randomized pilot intervention comparing the effect of intermittent energy restriction combined with a Mediterranean diet (IER+MED) to a euenergetic Dietary Approaches to Stop Hypertension (DASH) diet, with matching physical activity regimens, for reducing visceral adipose tissue area (VAT) and weight.

Methods: We analyzed the 59 (98\%) participants who completed at least one week of HDLS. Self-rated adherence scores to dietary and physical activity prescriptions were collected 8 times across the 12-week intervention, using a $0-10$ scale $(0=$ Not at all, $4=$ Somewhat, and $10=$ Following the plan very well). Adherence scores for each participant were averaged and assigned to high and low adherence categories using the group median (7.3 for diet, 7.1 for physical activity). Mean changes in VAT and weight from baseline to 12 weeks are reported by adherence level, overall and by randomization arm. Participants qualitative feedback at completion of HDLS and 6 months post intervention were examined.

Results: Overall, compared to participants with low self-rated adherence to dietary prescriptions, those with high adherence lost significantly more VAT $\left(22.9 \pm 3.7 \mathrm{~cm}^{2}\right.$ vs. $\left.11.7 \pm 3.9 \mathrm{~cm}^{2} ; p=0.044\right)$ and weight at Week 12 (5.4 $\pm 0.8 \mathrm{~kg}$ vs. $3.5 \pm 0.6 \mathrm{~kg} ; p=0.045)$. For physical activity, compared to participants with low adherence, those with high adherence lost significantly more VAT $\left(22.3 \pm 3.7 \mathrm{~cm}^{2}\right.$ vs. $11.6 \pm 3.6 \mathrm{~cm}^{2}$; $p=0.035)$. Participants' qualitative feedback of HDLS was positive and the most common response, on how to improve the study, was to provide cooking education classes.

Conclusions: Our results support the use of self-rated adherence to dietary and physical activity prescriptions as an effective method to monitor compliance and facilitate participant goal setting during a behavioral modification intervention. Study strategies were found to be effective with promoting compliance to dietary and physical activity prescriptions. The incorporation of cooking classes and demonstrations into future larger trials may further complement dietary adherence.

Trial registration: ClinicalTrials.gov Identifier: NCT03639350. Registered $21^{\text {st }}$ August 2018Retrospectively registered, https://clinicaltrials.gov/ct2/show/NCT03639350

\section{Introduction}

Overweight and obesity are pervasive risk factors for many non-communicable diseases (1). In particular, excess visceral adipose tissue (VAT) is associated with increased risk of cardio-metabolic disease, coronary artery calcification, type 2 diabetes, metabolic syndrome, certain cancers and non-alcoholic fatty liver disease (2-7). Adherence to lifestyle modification programs is known to be difficult $(8 ; 9)$; however, greater adherence is associated with improved obesity outcomes (9-14). No known study has assessed the association between intervention adherence and VAT loss. 
Research on lifestyle interventions aimed at reducing VAT have primarily been quantitative in nature (1519). However, a mixed-methods approach, using both quantitative and qualitative data, would help provide a more complete picture of study effectiveness, including participants' perspectives (20). This is especially important in nutrition interventions aimed at changing behaviors (20), and may assist in identifying factors influencing study adherence.

Previously our team reported the primary results of the randomized Healthy Diet and Lifestyle study (HDLS) pilot (21), aimed at reducing VAT among East Asian American adults. VAT and weight decreased in both study arms, but significantly more in the IER + MED group than the DASH group at 12 weeks (21). This secondary analysis aims to build on the primary analysis by assessing the utility of a self-rated scale used to monitor participants' dietary and physical activity compliance and facilitate goal setting during HDLS. We also aim to evaluate HDLS by analyzing participants' qualitative feedback collected upon completion and at 6-months post intervention.

\section{Methods}

\section{Study design}

The HDLS pilot study was a 12-week randomized trial conducted at the University of Hawaii Cancer Center (UHCC) between September 2016 and October 2017 to demonstrate feasibility and efficacy of following the IER+MED diet as an approach to reduce VAT. Extensive details of the intervention are provided in a previous publication (21). Briefly, eligibility included being of East Asian ancestry (Japanese, Chinese or Korean), residing in Honolulu County, BMI between 25 and $40 \mathrm{~kg} / \mathrm{m}^{2}$, ages 35 to 55 years, no pregnancy, and no serious health issues. Inclusion criteria included normal blood count and biochemistry profile and whole-body dual-energy $x$-ray absorptiometry (DXA)-derived VAT at L4-L5 $\geq 90 \mathrm{~cm}^{2}$ for men and $\geq 80 \mathrm{~cm}^{2}$ for women as determined at the eligibility clinic visit.

HDLS included baseline and Week 12 measurements of anthropometry and DXA (21). For the current analysis, outcomes of interest include body weight and VAT. One participant was excluded from analyses as s/he dropped out during the first week of HDLS and, thus, had no self-rated adherence data.

The study protocol [NCT03639350] was approved by the institutional review board at the University of Hawaii at Manoa. Study volunteers provided written informed consent. Figure 1 is a CONSORT diagram and the CONSORT and TIDieR checklists are provided as supplementary material (Additional file 1 and 2 , respectively).

\section{Randomization and masking}

Once eligibility was confirmed, stratified and blocked randomization was used to allot participants into either the IER+MED $(n=30)$ or DASH $(n=30)$ group, to ensure balance between groups. Randomization strata were defined by sex and high $\left(80 \mathrm{~cm}^{2}\right.$ for women or $90 \mathrm{~cm}^{2}$ for men to $\left.<150 \mathrm{~cm}^{2}\right)$ or very high 
$\left(\geq 150 \mathrm{~cm}^{2}\right)$ VAT. The assigned diets were labeled as either Diet A or Diet 1 to blind participants, recruitment, and clinic staff to IER+MED or DASH group allocation. Research dietitians, who were responsible for intervention activities, were blinded to participant measures except diet and body weight.

\section{Procedures and outcomes}

\section{Dietary and physical activity prescriptions}

Diet and physical activity prescriptions in HDLS have been reported previously (21). Briefly, the intervention group was assigned a IER+MED diet, and the active comparator group was assigned a euenergetic DASH diet for 12 weeks. Dietary materials for IER+MED were originally developed and tested for use among white women in the United Kingdom (15) and were modified to include examples of foods and beverages more readily available in Hawaii (21). Both groups were advised to walk one hour daily, five days per week, to reduce confounding due to physical activity. Participants in the IER+MED group were advised to exercise only on their MED days.

During an in-person dietary consultation ( 45-60 minutes) participants were provided with a personalized, group-specific, diet booklet (depicting serving sizes within food groups and examples of foods), individualized food lists and menus, and trackers to encourage compliance to prescriptions. Dietitians called participants once a week between Weeks 1 and 4, and at Week 6, 8 and 10, and conducted an in person follow-up at Week 12, to assess participants' adherence to their intervention plans (22) and provide guidance using motivational interviewing principles $(23 ; 24)$.

\section{Assessment of adherence to prescribed diet and physical activity prescriptions}

During follow-up calls conducted by study dietitians and the in-person dietitian consultation at Week 12, all participants were asked: "How well have you been following your diet plan? On a scale of zero to ten with zero being not at all, four being somewhat, and ten being following the plan very well, where would you place yourself?". Similarly, the same questions were asked for adherence to physical activity prescriptions. Self-rated adherence scores assisted with identifying participant's barriers to change and setting personal diet and physical activity goals to achieve by the next follow-up call $(23 ; 25)$.

These assessment questions were adapted from those used to assess motivation and confidence to change dietary behaviors used by Resnicow et al. $(23 ; 24 ; 26)$. For example, in the Body \& Soul Study, participants were asked "on a scale of 1 to 10 (with 10 being the highest), how motivated or interested are you in increasing your fruit and vegetable consumption?" Resnicow et al. found that this time and costeffective assessment technique assisted with increasing fruit and vegetable intake among African Americans in a church setting. 
Responses to self-rated adherence scores across the 12 week HDLS were averaged for each person, and participants were divided into a high or low level of adherence, split by median score (7.3 for diet and 7.1 for physical activity).

\section{Qualitative Feedback}

During the clinic visit at Week 12, participants completed a self-administered exit questionnaire. The two questions relevant to this analysis were if he/she would have been interested in cooking classes or demonstrations and whether the materials provided were sufficient to be able to follow the diet plan. Participants recorded their responses using a 5-point Likert scale (strongly agree/ agree/ neither agree or disagree/ disagree/ strongly disagree).

At 6-months post-intervention, recruitment staff performed a follow-up, by telephone, of participants who completed the HDLS. Participants were interviewed using a standardized questionnaire tailored to the study objectives. Questions pertinent to this analysis included "What type or types of exercise have you been doing since you completed the study?"; "Are you still trying to follow the diet you were assigned to during the study?"; "If no, can you describe the type of diet that you have been following since you completed the study?"; and "Do you have any comments or suggestions as to how the study could be improved?".

\section{Statistical analysis}

Analyses were limited to participants with complete Week 1 study data $(n=59)$. Multiple imputation was used to replace missing values of the outcome and exposure variables and generate five imputed datasets. Less than $5 \%$ of data values were missing. Mean changes in VAT and weight loss, from baseline to 12 weeks, were computed by self-reported adherence level, overall and by randomization arm, and compared between adherence levels using a t-test. Responses to 5-point Likert scale questions are reported as frequencies and percentages. Quantitative analyses were performed using IBM SPSS Statistics version 26 (IBM Corp., Armonk, NY, USA) and statistical significance was defined as $p<0.05$.

Qualitative methods were used to evaluate responses to the open-ended 6-month post-intervention telephone interview questions (27). Responses to open-ended questions from each participant were transcribed separately by staff members not involved in the study. Co-investigators (KC, HO) and another staff member independently coded each response transcript. Each coder identified and nominated common themes and preliminary codes, and all codes were reviewed and discussed by the team until a final consensus was reached. A codebook was then developed for analysis using NVIVO Version 11 (QSR International, Melbourne, Australia) and used to drive a subsequent thematic analysis of all interview transcript data (27).

\section{Results}




\section{Characteristics}

Of the 60 participants enrolled in HDLS, six (10\%) dropped out (IER+MED:4; DASH:2), with only one participant withdrawing from the study before the Week 1 follow-up call. Of the 54 participants completing HDLS, all 54 participants completed the Exit Questionnaire and 48 participants responded to the six-month post-intervention telephone interview. Of participants included in the current analysis, 29 (48.2\%) were randomized to IER+MED and $30(50.8 \%)$ to DASH (Table 1$)$. The study participants were mostly women (69.5\%), participants with high VAT (67.8\%), and of Japanese ancestry (62.7\%). Baseline characteristics were similar for the 59 participants included in this analysis, the 54 participants who completed the Week 12 visit and the 48 who completed the 6-month post-intervention survey.

Splitting data by median adherence, baseline characteristics were similar between dietary adherence groups and between physical activity adherence groups (Table 1). The largest differences in adherence were seen between ethnic groups. For dietary adherence, $71.4 \%(n=5)$ participants of Korean or Mixed Asian ancestry were in the low category. For physical activity adherence, $100 \%(n=7)$ of participants with Korean ancestry were in the low group, and $75 \%(n=6)$ of participants of Chinese ancestry were in the high adherence group. 
Table 1

Baseline characteristics of participants $(n=59)$ by self-rated adherence to dietary and physical activity prescriptions

\begin{tabular}{|c|c|c|c|c|c|}
\hline \multirow[b]{2}{*}{ Characteristic } & \multirow[b]{2}{*}{ All } & \multicolumn{2}{|c|}{ Dietary adherence ${ }^{1,2}$} & \multicolumn{2}{|c|}{$\begin{array}{l}\text { Physical activity } \\
\text { adherence } e^{2,3}\end{array}$} \\
\hline & & Low & High & Low & High \\
\hline \multicolumn{6}{|l|}{ Study arm, $n(\%)^{4}$} \\
\hline IER+MED 5 & $29(49.2)$ & $14(48.3)$ & $15(51.7)$ & $12(41.4)$ & $17(58.6)$ \\
\hline $\mathrm{DASH}^{6}$ & $30(50.8)$ & $17(56.7)$ & $13(43.3)$ & $16(53.3)$ & $14(46.7)$ \\
\hline \multicolumn{6}{|l|}{ Sex, $n(\%)$} \\
\hline Men & $18(30.5)$ & $7(38.9)$ & $11(61.1)$ & $8(44.4)$ & $10(55.6)$ \\
\hline Women & $41(69.5)$ & $24(58.5)$ & $17(41.5)$ & $20(48.8)$ & $21(51.2)$ \\
\hline \multicolumn{6}{|c|}{ Visceral adipose tissue, $n(\%)$} \\
\hline $\begin{array}{l}\mathrm{High}^{7} \text { (80 or } 90 \text { to }<150 \\
\mathrm{~cm}^{2} \text { ) }\end{array}$ & $40(67.8)$ & $23(57.5)$ & $17(42.5)$ & $20(50.0)$ & $20(50.0)$ \\
\hline Very high $\left(\geq 150 \mathrm{~cm}^{2}\right)$ & $19(32.2)$ & $8(42.1)$ & $11(57.9)$ & $8(42.1)$ & $11(57.9)$ \\
\hline \multicolumn{6}{|l|}{ Ethnicity, $n(\%)$} \\
\hline Chinese & $8(13.6)$ & $4(50.0)$ & $4(50.0)$ & $2(25.0)$ & $6(75.0)$ \\
\hline Japanese & $37(62.7)$ & $17(45.9)$ & $20(54.1)$ & $15(40.5)$ & $22(59.5)$ \\
\hline Korean & $7(11.9)$ & $5(71.4)$ & $2(28.6)$ & $7(100.0)$ & $0(0.0)$ \\
\hline Mixed Asian & $7(11.9)$ & $5(71.4)$ & $2(28.6)$ & $4(57.1)$ & $3(42.9)$ \\
\hline \multicolumn{6}{|c|}{$\begin{array}{l}\text { Other Characteristics, mean } \pm \\
\text { SD }\end{array}$} \\
\hline Age, y & $47.4 \pm 5.1$ & $46.1 \pm 5.3$ & $48.9 \pm 4.4$ & $45.9 \pm 5.7$ & $48.9 \pm 4.0$ \\
\hline Weight, kg & $\begin{array}{l}80.4 \pm \\
12.4\end{array}$ & $\begin{array}{l}78.2 \pm \\
11.4\end{array}$ & $\begin{array}{l}82.8 \pm \\
13.4\end{array}$ & $80.0 \pm 11.3$ & $80.7 \pm 13.4$ \\
\hline Body mass index, $\mathrm{kg} / \mathrm{m}^{2}$ & $30.7 \pm 3.4$ & $30.2 \pm 3.4$ & $31.2 \pm 3.3$ & $30.5 \pm 3.6$ & $31.0 \pm 3.1$ \\
\hline
\end{tabular}

${ }^{1}$ Self-rated dietary adherence ranging from zero being not at all to ten being following the plan very well, split by median adherence (7.3). ${ }^{2}$ Imputed values for missing adherence data. ${ }^{3}$ Self-rated physical activity adherence ranging from zero being not at all to ten being following the plan very well, split by median adherence (7.1). ${ }^{4}$ Column percentages for overall column and row percentages for data by self-rated adherence level. ${ }^{5}$ Intermittent energy restriction combined with a Mediterranean diet 
(IER+MED). ${ }^{6}$ Euenergetic Dietary Approaches to Stop Hypertension diet (DASH). ${ }^{7}$ Women at $\geq 80 \mathrm{~cm}^{2}$ and men at $\geq 90 \mathrm{~cm}^{2}$.

\section{Adherence}

Overall, mean $\pm \mathrm{SE}$, dietary adherence over 12 weeks was $6.0 \pm 0.2$ and $8.2 \pm 0.1$, for the low and high adherence groups, respectively. Range of dietary adherence scores were 2.9-7.7 and 7.1-9.9 respectively. For physical activity adherence, mean scores were $5.9 \pm 0.2$ and $8.5 \pm 0.2$ for the low and high adherence groups, and ranged from 3.0-7.2 and 7.0-10.0, respectively.

Compared to participants with low self-rated adherence to dietary prescriptions, those with high adherence lost significantly more VAT $\left(22.9 \pm 3.7 \mathrm{~cm}^{2}\right.$ vs. $\left.11.7 \pm 3.9 \mathrm{~cm}^{2} ; p=0.044\right)$ and weight at Week 12 (5.4 $\pm 0.8 \mathrm{~kg}$ vs. $3.5 \pm 0.6 \mathrm{~kg} ; p=0.045$ ) (Table 2). For physical activity, compared to participants with low adherence, those with high adherence lost significantly more VAT $\left(22.3 \pm 3.7 \mathrm{~cm}^{2}\right.$ vs. $11.6 \pm 3.6 \mathrm{~cm}^{2}$; $p=0.035$ ) (Table 3). Weight loss was also greater for those with high vs. low adherence to physical activity prescriptions (5.0 $\pm 0.7 \mathrm{~kg}$ vs. $3.7 \pm 0.7 \mathrm{~kg})$; however, these differences were not significant. Within study arm comparisons, high dietary adherence and high physical activity adherence had greater VAT and weight loss than their counterpart low adherence groups, but these differences were not significant (Tables 2 and 3). Repeating analyses using \% change in VAT and \% change in weight instead of absolute change in VAT and weight produced similar results.

Table 2

Change in visceral adipose tissue (VAT) and weight by self-rated adherence to dietary prescriptions ${ }^{1}$

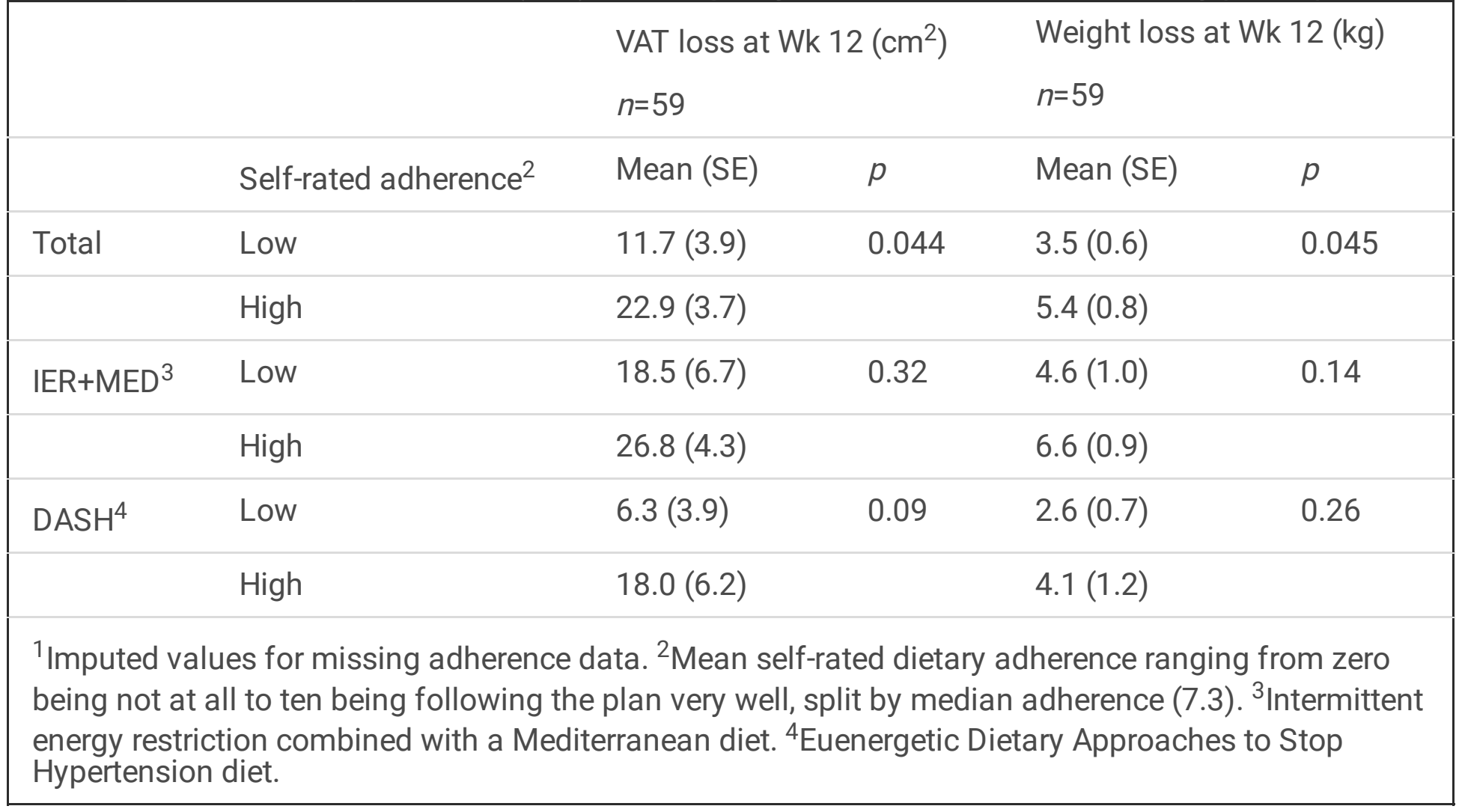


Table 3

TABLE 3. Change in visceral adipose tissue (VAT) and weight by self-rated adherence to physical activity prescriptions ${ }^{1}$

\begin{tabular}{|c|c|c|c|c|c|}
\hline & \multirow[b]{2}{*}{ Self-rated adherence ${ }^{2}$} & \multicolumn{2}{|c|}{$\begin{array}{l}\text { VAT loss at Wk } 12\left(\mathrm{~cm}^{2}\right) \\
\qquad n=59\end{array}$} & \multicolumn{2}{|c|}{$\begin{array}{l}\text { Weight loss at Wk } 12(\mathrm{~kg}) \\
n=59\end{array}$} \\
\hline & & Mean (SE) & $p$ & Mean (SE) & $p$ \\
\hline \multirow[t]{2}{*}{ Total } & Low & $11.6(3.6)$ & 0.035 & $3.7(0.7)$ & 0.20 \\
\hline & High & $22.3(3.7)$ & & $5.0(0.7)$ & \\
\hline \multirow[t]{2}{*}{ IER+MED 3} & Low & $17.8(5.9)$ & 0.20 & $5.5(1.1)$ & 0.86 \\
\hline & High & $26.8(4.4)$ & & $5.8(0.9)$ & \\
\hline \multirow[t]{2}{*}{$\mathrm{DASH}^{4}$} & Low & $6.8(4.1)$ & 0.15 & $2.5(0.8)$ & 0.18 \\
\hline & High & $16.8(5.8)$ & & $4.2(1.0)$ & \\
\hline
\end{tabular}

${ }^{1}$ Imputed values for missing adherence data. ${ }^{2}$ Mean self-rated physical activity adherence ranging from zero being not at all to ten being following the plan very well, split by median adherence (7.1). ${ }^{3}$ Intermittent energy restriction combined with a Mediterranean diet. ${ }^{4}$ Euenergetic Dietary Approaches to Stop Hypertension diet.

\section{Qualitative Feedback}

Answering the question, "materials provided were sufficient to be able to follow the diet plan", 44 (81\%) participants rated that they strongly agree or agree, 8 (15\%) that they neither agree or disagree, and 2 (4\%) participants did not respond. For the question "Would in-house classes or cooking demonstrations have been of interest?" 36 (67\%) participants answered yes, 12 (22\%) reported no, 2 (4\%) were undecided, and $4(7 \%)$ did not answer the question. Women tended to be more interested in the in-house classes or cooking demonstrations than men ( $75 \%$ and $47 \%$, respectively).

The thematic structure identified for the open-ended questions as part of the 6-month post-intervention telephone interview followed the topics of the survey questions including 1) Exercise; 2) Diets; 3) Comments; and 4) Suggestions (27). Participant responses were summarized based on this thematic structure.

Exercise. Almost all ( $n=46,96 \%)$ participants reported continuing exercise at 6-months post-intervention. Overall, respondents reported 60 types of exercise regimens they adopted or continued after the study, with 31 (65\%) reporting "walking" as a primary form of exercise. Other popular exercise included running, swimming, paddling, tennis, golf, cycling, weightlifting, fishing, Zumba, Step Aerobics, Aqua Aerobics, high intensity interval training, calisthenics, stair climbing, and use of a gymnasium.

Diets. Approximately half of the respondents reported "yes", they were still trying to follow their prescribed diet at 6-months post-intervention (IER+MED, 66.7\%; DASH, 44.0\%). Of those participants not following the diet at 6-months post-study, many $(n=8)$ reported focusing on a healthy diet without mentioning the specific dietary changes. For example, "Your study increased my awareness regarding foods and I am eating healthier, and use the basics of the study as a guide." Also, although no longer following their 
prescribed diet, participants reported substituting nutrient poor items for nutrient dense choices $(n=4)$, reducing their portion size $(n=9)$, decreasing intake of sugar, meat or carbohydrates $(n=7)$, and increasing intake of fruit and vegetables ( $n=14)$. An example being, "gave up some snack foods and unhealthy food. More aware of healthier options." In addition, participants reported adopting a modified version of the study diet with the re-adoption of non-study foods $(n=6)$, for example, "following protocol, but added dark chocolate."

Comments. Under this theme, praise for the support of the intervention dietitians was the most frequent comment $(n=14)$. For example, "Helpful to have dietitian suggestions and accountability." Unfamiliarity with the foods prescribed during the study, and preparation of these foods $(n=2)$ was the second most frequent comment.

Suggestions. The most common response, on how to improve the study, was to provide cooking education $(n=8)$ in the form of classes delivered either in-person or online; and the creation of a cookbook to support the study. For example, "would really appreciate cooking classes." The Identification of more varied foods to support the study was also suggested, including the identification of premade foods $(n=3)$ that would be acceptable for use, and longer study duration $(n=1)$.

\section{Discussion}

Among all participants, higher self-rated adherence to dietary or physical activity prescriptions was associated with significantly greater loss of VAT on completion of the 12-week HDLS pilot. This is the first known randomized trial to report an association between self-rated adherence and VAT loss. The association found between dietary adherence and weight loss supports previous findings, with higher adherence resulting in greater loss $(10 ; 14)$.

The single-item questions used to assess adherence in HDLS were adapted from questions used by Resnicow et al $(23 ; 24)$. Resnicow et al. demonstrated that these single-item questions, based on motivational interviewing, were effective at evaluating confidence and motivation to change, and for eliciting motivational messages and barriers to change $(23 ; 24 ; 26)$. Similarly, Dansinger et al used a $0-10$ scale to assess participants self-rated adherence and the effectiveness of 4 popular diets for weight loss (10). In both the current analysis and the Dansigner et al study, greater dietary adherence was associated with weight loss. These results support a single-item question can be used to assess intervention compliance and assist with facilitating behavior change during an intervention.

Several other studies have verified the agreement between self-evaluation and behavior. A cross-sectional study by Adjoian et al. assessed the validity of self-rated overall diet quality compared to Healthy Eating Index-2010 (HEl-2010) scores among a multiethnic adult population in New York City (NYC) (28). Those with lower self-rated diet quality had significantly lower HEl-2010 scores. In regards to physical activity, a single-item questionnaire, assessing participants activity in the previous week, has been validated against accelerometer data (29). The question asks "In the past week, on how many days have you done a total of 30 min or more of physical activity, which was enough to raise your breathing rate? This may include 
sport, exercise and brisk walking or cycling for recreation or to get to and from places, but should not include housework or physical activity that may be part of your job". These studies add to the value of short self-report questionnaires, where participant responses matched direct objective measures of study outcomes. However, the single-item questions utilized in HDLS are unique in that the questions are broad; therefore, theoretically, they could be applied to assess self-rated compliance to a range of intervention prescriptions.

Key strategies for dietary adherence include designing weight loss studies that limit hunger, are tailored to participants dietary preferences, and promote self-monitoring of dietary intake (9). All these strategies were employed in HDLS (21), which may explain why both low and high dietary adherence groups successfully lost VAT and weight, mean dietary adherence for both study arms was relatively high (IER + MED, 7.3; DASH, 6.8), and participants' positive reflections of the study. However, modifications to the design of HDLS may help to further improve dietary adherence. For example, responses to both the Exit Questionnaire and 6-month post-study telephone interview indicated that most participants $(\sim 2 / 3)$ were interested in cooking classes and demonstrations. The integration of these classes may help to further personalize dietary prescriptions, reduce any unfamiliarity of foods prescribed, increase participants confidence in preparing meals (30), and ultimately improve study compliance, long-term adherence and adoption.

HDLS participants did not provide any comments or suggestions on how to improve physical activity prescriptions. However, ethnicity appeared to be a large driver for physical activity adherence, with all participants of Korean ancestry $(n=7)$ being in the low adherence category. A review on adherence to lifestyle modifications programs for weight management highlights strategies which may help improve physical activity compliance for those in the low adherence category (9). The authors identified psychosocial factors, socio-demographic factors, behavioral factors, and physical factors as influencing adherence. For example, psychological factors influencing adherence include self-efficacy, depression, motivation, stress, body shape concern, quality of life and stage of change. Socio-demographic factors include age, gender, employment status and education (9). Focusing on reasons for ethnic differences in physical activity adherence and additional predictors of adherence, may help to improve physical activity compliance scores in future trials.

The strengths of the current study include the evidence-based strategies implemented to ensure participant engagement and compliance. These include the adoption of dietary protocols by Harvie et al (15), and behavior change strategies from Body and Soul $(23 ; 24)$. A limitation was the small sample size which may have reduced statistical power to show significant associations between adherence and changes in VAT and body weight by study arms. Also, the association seen between weight loss and adherence level may have been due to reverse causation. Participants with greater weight loss may have thought they were adhering to the dietary and physical activity prescriptions more closely, and rated their adherence higher. However, change in VAT is harder for participants to self-monitor and change in VAT between adherence arms was proportionately larger than change in weight. Therefore, it is unlikely VAT results were due to reverse causation. Another limitation is change in VAT and weight were used as proxy 
measurements for adherence to intervention prescriptions. To assess the validity of self-rated compliance to monitor intervention adherence, future studies should compare self-rated adherence results to more direct objective measurements as opposed to proxy methods (e.g., compare self-rated physical activity adherence to accelerometer data).

\section{Conclusions}

Results support the utility of self-rated compliance as a method to monitor dietary and physical activity adherence and facilitate participant goal setting during a behavioral modification intervention aimed at reducing VAT and weight. Overall, participant feedback on HDLS was positive, and incorporation of cooking classes and demonstrations into future larger trials may help to further improve dietary adherence.

\section{Abbreviations}

BMI

Body Mass Index

DASH

Dietary Approaches to Stop Hypertension

DXA

Dual energy $\mathrm{x}$-ray absorptiometry

HDLS

Healthy Diet and Lifestyle Study

$\mathrm{IER}+\mathrm{MED}$

Intermittent energy restriction combined with a Mediterranean diet

UHCC

University of Hawaii Cancer Center

VAT

Visceral adipose tissue

\section{Declarations}

\section{Ethics approval and consent to participate}

All study procedures were approved by the University of Hawaii Institutional Review Board and written informed consent was obtained from all study participants.

\section{Consent for publication}

Not applicable 


\section{Availability of data and materials}

The datasets used and/or analyzed during the current study are available from the corresponding author on reasonable request.

\section{Competing interests}

The authors declare that they have no competing interests.

\section{funding}

This research was funded by the William \& Ellen Melohn Endowed Research Fund, University of Hawaii; and supported in part by the National Cancer Institute at the National Institutes of Health grants P30 CA071789. Michelle Harvie is supported by the National Institute for Health Research (NIHR) Manchester Biomedical Research Centre.

\section{Authors contributions}

L.L.M. conceived the Healthy Diet and Lifestyle Study; C.J.B, K.D.C., K.M.Y., L.L.M., L.R.W., M.N.H., and U.L., collaborated on the design of the Healthy Diet and Lifestyle Study; C.E.P., C.J.B, H.O., and K.D.C., analyzed the data; C.E.P., C.J.B., F.M.Z., H.O., J.A.S., K.D.C., K.M.Y., L.L.M., L.R.W., M.N.H., U.L., and Y.B.S., assisted with data analysis and interpretation of results, and wrote the manuscript.

\section{Acknowledgements}

We would like to thank study participants for their participation. From the University of Hawaii Cancer Center, we thank the Nutrition Support Shared Resource including Jean Ishida for assisting with development of dietary materials, intervention activities, and data entry; the Biostatistics Shared Resource for support with data analyses and development of the randomization protocol; Lucia Amore, Anthony Reedy, and Shirley So, for coordination and collection of clinic measurements; Naomi Hee and Clara Richards for eligibility screening, coordinating study visits, and conducting six month post-study followup calls; Terrilea Burnett for assistance with eligibility screening and study coordination; John Shepherd's lab for conducting study measurements, quality control and data cleaning, and Nana Ohkawa for public relations support.

\section{References}

1. World Health Organization. Obesity and Overweight. [cited 2020 May 28]. Available from: https://www.who.int/news-room/fact-sheets/detail/obesity-and-overweight. 
2. Liesenfeld DB, Grapov D, Fahrmann JF, Salou M, Scherer D, Toth R, et al. Metabolomics and transcriptomics identify pathway differences between visceral and subcutaneous adipose tissue in colorectal cancer patients: the ColoCare study. Am J Clin Nutr. 2015;102:433-43.

3. Needland IJ, Turer AT, Ayers CR, Powell-Wiley TM, Vega GL, Farzaneh-Far R, et al. Dysfunctional adiposity and the risk of prediabetes and type 2 diabetes in obese adults. JAMA. 2012;19(308):1150-9.

4. Oliveira CP, de Lima Sanches P, de Abreu-Silva EO, Marcadenti A. Nutrition and physical activity in nonalcoholic fatty liver disease. J Diabetes Res. 2016; 2016(4597246): 1-12.

5. Kang YM, Jung CH, Cho YK, Jang JE, Hwang JY, Kim EH, et al. Visceral adiposity index predicts the conversion of metabolically healthy obesity to an unhealthy phenotype. PLoS One. 2017;12(6):e0179635.

6. MassI R, van Blankenstein M, Jeurnik S, Hermans J, de Haan M, Stoker J. Visceral adipose tissue: the link with esophageal adenocarcinoma. Scand J Gastroenterol. 2014;49(4):449-57.

7. Shah RV, Murthy VL, Abbasi SA, Blankstein R, Kwong RY, Goldfine AB, et al. Visceral adiposity and the risk of metabolic syndrome across body mass index: the MESA Study. JACC Cardiovasc Imaging. $2014 ; 7(12): 1221-35$.

8. Jensen MD, Ryan DH, Apovian CM, Ard JD, Comuzzie AG, Donato KA, et al. 2013 AHA/ACC/TOS guideline for the management of overweight and obesity in adults: A report of the American College of Cardiology/American Heart Association Task Force on Practice Guidelines and The Obesity Society. J Am Coll Cardiol. 2014;63:2985-3023.

9. Leung AWY, Chan RSM, Sea MMM, Woo J. An overview of factors associated with adherence to lifestyle modification programs for weight management in adults. Int J Environ Res Public Health. 2017; 14(922).

10. Dansinger ML, Gleason J, Griffith JL, Selker HP, Schaefer EJ. Comparison of the Atkins, Ornish, Weight Watchers, and Zone diets for weight loss and heart disease risk reduction: A randomized trial. JAMA. 2005;293:43-53.

11. Wadden TA, West DS, Neiberg RH, Wing RR, Ryan DH, Johnson KC, et al. One-year weight losses in the Look AHEAD study: Factors associated with success. Obesity. 2009;17:713-22.

12. Gibson AA, Sainsbury A. Strategies to improve adherence to dietary weight loss interventions in research and real-world settings. Behav Sci. 2017; 7(44).

13. Bartfield JK, Stevens VJ, Jerome GJ, Batch BC, Kennedy BM, Vollmer WM, et al. Behavioral transitions and weight change patterns within the PREMIER trial. Obesity. 2011;19:1609-15.

14. Acharya SD, Elci OU, Sereika SM, Music E, Styn MA, Turk MW, et al. Adherence to a behavioral weight loss treatment program enhances weight loss and improvements in biomarkers. Patient Preference Adherence. 2009;3:151-60.

15. Harvie M, Wright C, Pegington M, McMullan D, Mitchell E, Martin B, et al. The effect of intermittent energy and carbohydrate restriction v. daily energy restriction on weight loss and metabolic disease risk markers in overweight women. Br J Nutr. 2013;110(8):1534-47. 
16. Harvie MN, Pegington M, Mattson MP, Frystyk J, Dillon B, Evans G, et al. The effects of intermittent or continuous energy restriction on weight loss and metabolic disease risk markers: a randomized trial in young overweight women. Int J Obes (Lond). 2011;35(5):714-27.

17. Schubel R, Nattenmuller J, Sookthai D, Nonnenmacher T, Graf ME, Riedl L, et al. Effects of intermittent and continuous calorie restriction on body weight and metabolism over $50 \mathrm{wk}$ : a randomized controlled trial. Am J Clin Nutr. 2018;108(5):933-45.

18. Conley M, Le Fevre L, Haywood C, Proietto J. Is two days of intermittent energy restriction per week a feasible weight loss approach in obese males? A randomised pilot study. Nutr Diet. 2017;75(1):6572.

19. Carter S, Clifton PM, Keogh JB. Effect of intermittent compared with continuous energy restricted diet on glycemic control in patients with type 2 diabetes: a randomized noninferiority trial. JAMA Netw Open. 2018;1(3):1-12.

20. Harris JE, Gleason PM, Sheean PM, Boushey C, Beto JA, Bruemmer B. An introduction to qualitative research for food and nutrition professionals. J Am Diet Assoc. 2009;109:80-90.

21. Panizza CE, Lim U, Yonemori KM, Cassel KD, Wilkens LR, Harvie MN, et al. Effects of intermittent energy restriction combined with a Mediterranean diet on reducing visceral adiposity: a randomized active comparator pilot study. Nutrients. 2019; 1-20.

22. Heymsfield SB, Wadden TA. Mechanisms, pathophysiology, and management of obesity. N Engl J Med. 2017;375:254-66.

23. Resnicow K, Jackson A, Wang T, De AK, McCarty F, Dudley WN, et al. A motivational interviewing intervention to increase fruit and vegetable intake through Black churches: results of the Eat for Life trial. Am J Public Health. 2001;91(10):1686-93.

24. Resnicow K, Campbell MK, Carr C, McCarty F, Wang T, Periasamy S, et al. Body and soul. A dietary intervention conducted through African-American churches. Am J Prev Med. 2004;27(2):97-105.

25. Rollnick S, Butler CC, Stott N. Helping smokers make decisions: the enhancement of brief intervention for general medical practice. Patient Educ Couns. 1997;31(3):191-203.

26. Resnicow K, Jackson A, Blissett D, Wang T, McCarty F, Rahotep S, et al. Results of the healthy body healthy spirit trial. Health Psychol. 2005;24(4):339-48.

27. Patton MQ. Qualitative research and evaluation methods. 3rd ed. London: Sage Publications; 2002.

28. Adjoin TK, Firestone MJ, Eisenhower D, Yi SS. Validation of self-rated overall diet quality by Healthy Eating Index-2010 score among New York City adults, 2013. Prev Med Rep. 2016;3:127-31.

29. Milton K, Clemes S, Bull F. Can a single question provide an accurate measure of physical activity? $\mathrm{Br}$ J Sports Med. 2013;47:44-8.

30. Hartmann C, Dohle S, Siegrist M. Importance of cooking skills for balanced food choices. Appetite. 2013;65:125-31.

\section{Figures}




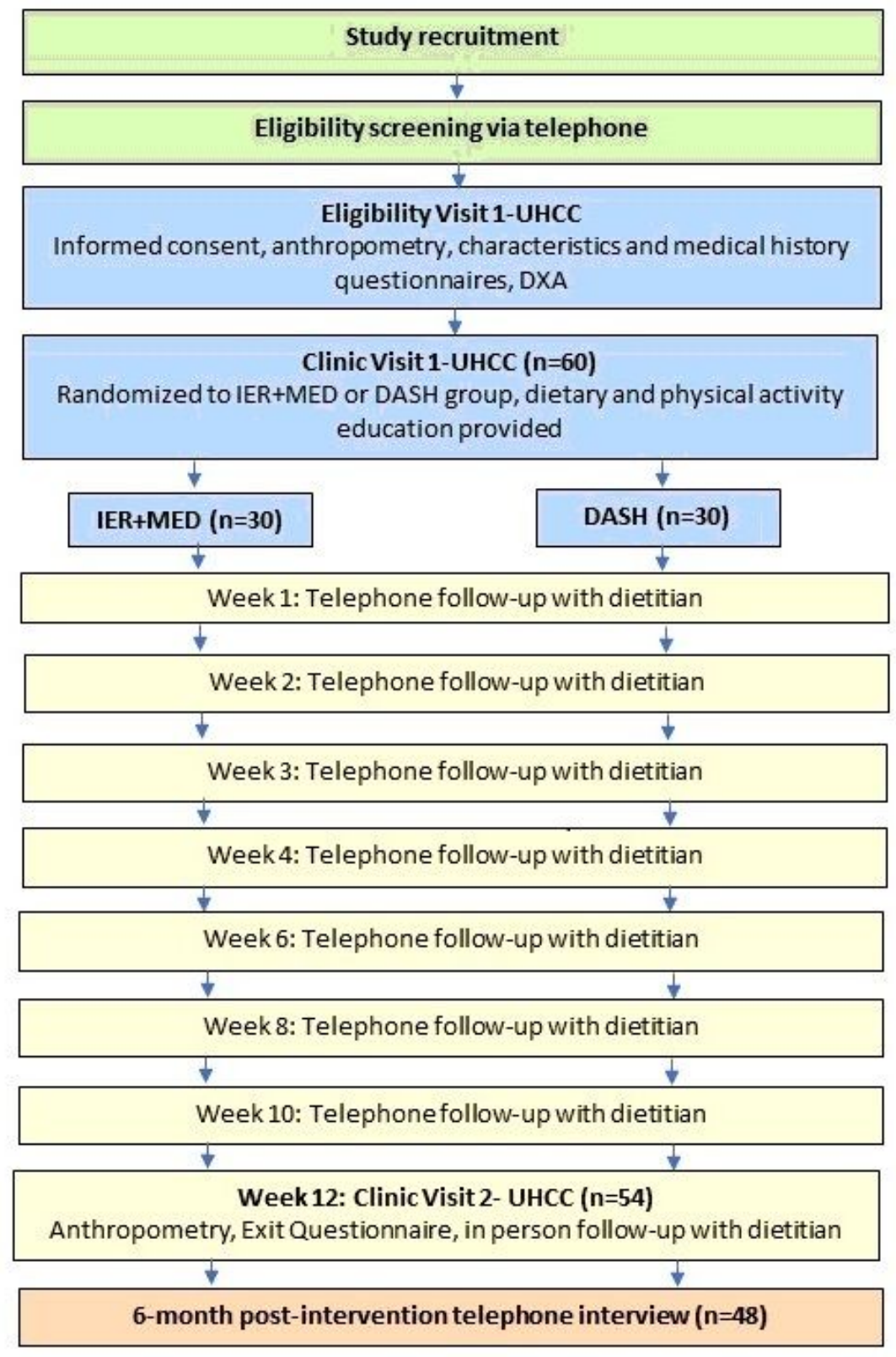

Baseline measurements, randomization 12-week intervention 6-months post-intervention

\section{Figure 1}

Healthy Diet and Lifestyle Study flow diagram

\section{Supplementary Files}

This is a list of supplementary files associated with this preprint. Click to download. 
- AdditionalFile2.docx

- AdditionalFile1.doc 